\title{
Research on Comprehensive Evaluation of Customer Satisfaction of Computer Products
}

\author{
Jimin Gao \\ Department of Computer Engineering, Shenzhen Polytechnic, Shenzhen, China \\ gaojm@szpt.edu.cn
}

Keywords: Computer products, Customer satisfaction, Fuzzy hierarchical analytical evaluation method

\begin{abstract}
Through the research on the characteristics of computer products, this paper constructs the customer satisfaction evaluation model of computer products. Based on the statistics and analysis of data, the evaluation of customer satisfaction of computer products is obtained by using fuzzy comprehensive evaluation method. On this basis, this paper analyzes how enterprises can improve customer satisfaction, and then the strategy and methods of winning more customers are gained, which provides a reference for enhancing the market competitiveness of computer enterprises in China.
\end{abstract}

\section{Introduction}

With the rapid development of the Internet economy, computers have become familiar to consumers, and computer products have become the necessities of life for many consumers. At the same time, the technology advances by leaps and bounds, the acceleration of computer product upgrading and the fierce market competition make the computer enterprise facing increasingly serious competition situation[1]. The company recognizes the importance of gaining market share, attracting and maintaining customers' continuous growth. Customer satisfaction is the key factor of winning the enterprise in the fierce market competition, the enterprise according to the analysis of the factors that affect computer products customer satisfaction to enhance the understanding of the market, thus more effectively improve customer satisfaction, help maintain customer loyalty to the enterprise, so as to improve enterprise's competitiveness[2].

\section{The Characteristics of Computer Products}

Based on the comprehensive analysis of existing literature, this paper studies computer products from the perspective of marketing, and concludes that computer products have the following characteristics[3][4]:

(1) The application subject is popular. The rapid development of network economy and continuous technology innovation promote the breakthrough of the computer industry, and make computer product prices keep falling. Computer products have been accepted by the broad masses of customers, and gradually become the necessities of consumers.

(2) Strong professional and implicit demand. For most consumers, with strong professional computer products, mostly are passively using existing function of computer products, but this does not mean that the consumer is not deep demand for computer products.

(3) Quick innovation and short life cycle. The continuous innovation of technology is the source of the development of high and new technology products, and the computer products are updated quickly and the life cycle becomes shorter.

(4) The price of the product has fallen fast. The trend of faster updating and shorter life cycles has led to a decline in the price of computer products.

\section{Design of Evaluation Index System for Customer Satisfaction of Computer Products}

In this paper, based on the customer satisfaction theory and the characteristics of computer products 
industry and consumer point of view, following the principle of customer subjectivity, overall principle, the materiality principle, operational principle, etc., the comprehensive evaluation index system for customer satisfaction of computer products is built, which is shown in Table 1.

Table. 1 Evaluation index system for customer satisfaction of computer products

\begin{tabular}{|c|c|c|}
\hline Target layer & Criterion layer & Index layer \\
\hline \multirow{4}{*}{$\begin{array}{c}\text { Customer } \\
\text { satisfaction of } \\
\text { computer products }\end{array}$} & $\begin{array}{c}\text { Product comprehensive } \\
\text { performance }\end{array}$ & Product function \\
\cline { 3 - 3 } & & The hardware configuration \\
\cline { 3 - 3 } & & The software design \\
\cline { 3 - 3 } & The enterprise image & Product safety \\
\cline { 3 - 3 } & \multirow{3}{*}{ The quality of service } & Enterprise appareness \\
\cline { 3 - 3 } & & Corporate reputation \\
\cline { 3 - 3 } & & Staff attitudes \\
\cline { 3 - 3 } & & Sales environment \\
\hline
\end{tabular}

\section{Comprehensive Evaluation for Customer Satisfaction of Computer Products based on Fuzzy Hierarchy}

The Basic Steps of Fuzzy Analytic Hierarchy Process. The basic steps of evaluating computer product customer satisfaction with fuzzy hierarchy process are as follows:

(1) Determine the evaluation target. Construct computer product customer satisfaction evaluation fuzzy set of construction machinery $V=\left\{v_{1}, v_{2}, v_{3}, v_{4}\right\}$, namely dividing product design into four levels. $v_{1}$ represents the excellent level, $v_{2}$ represents the good level, $v_{3}$ represents the medium level and $v_{4}$ represents the bad level.

(2) Establish the evaluation index system. Determine the field of factors influenced computer product customer satisfaction $U=\left\{U_{1}, U_{2}, U_{3}\right\}, U_{1}$ represents the product comprehensive performance in criterion layer belonging to first level fuzzy evaluation. According to the sub-index, divide the first level evaluation indexes into $U_{i}=\left\{u_{1}^{(i)}, u_{2}^{(i)}, \cdots, u_{j}^{(i)}\right\}$, where $u_{j}^{(i)}$ corresponds each element of index layer belonging to second level fuzzy evaluation, $i$ and $j$ respectively represents the number of evaluation indexes in criterion layer and corresponding index layer.

(3) Construct fuzzy judgment matrix. Based on the evaluation index system, search the affiliation of computer product customer satisfaction evaluation level $v_{m}(m=1,2,3,4)$ from evaluation indexes. Then construct the affiliation function and calculate the affiliation degree. Each element of index layer may establish a fuzzy judgment vector: $R=\left\{r_{11}^{(i)}, r_{12}^{(i)}, r_{13}^{(i)}, r_{14}^{(i)}\right\}$. And all the single fuzzy judgment vector can make up the fuzzy judgment matrix:

$$
R_{i}=\left[\begin{array}{llll}
r_{11} & r_{12} & r_{13} & r_{14} \\
r_{21} & r_{22} & r_{23} & r_{24} \\
r_{31} & r_{32} & r_{33} & r_{34}
\end{array}\right]
$$

In single evaluation matrix, each row represents a degree of membership of the respective computer product customer satisfaction factors of four levels of product design evaluation, and the column indicates the number of computer product customer satisfaction factors.

(4) Determine the weight value. In the process of computer product customer satisfaction evaluation, each factor make a different important influence to the elements of upper level. Thus, it is necessary to set a weight value to each factors. For criterion layer, the three factors' weight are set as $A=\left\{A_{1}, A_{2}, A_{3}\right\}$, where $0 \leq A_{i} \leq 1, \sum_{i=1}^{3} A_{i}=1$. Similarly, for index layer, factors weights are set 
as $A_{i}=\left\{a_{1}^{(i)}, a_{2}^{(i)}, \cdots, a_{t}^{(i)}\right\}$, and $0 \leq a_{j}^{(i)} \leq 1, \quad \sum_{i=1}^{t} a_{i}=1$.

(5) Construct fuzzy hierarchy analysis model. The index weight of index layer $A_{i}=\left\{a_{1}^{(i)}, a_{2}^{(i)}, \cdots, a_{t}^{(i)}\right\}$ and fuzzy evaluation matrix $R_{i}$ is already known. Apply the weighted average operator to calculate the evaluation results of all single factors in criterion layer, which are shown as follow:

$$
B_{i}=A_{i} \circ R_{i}=\left(\begin{array}{llll}
b_{i 1} & b_{i 2} & \cdots & b_{i m}
\end{array}\right) \quad i=1,2, \cdots, t
$$

Where $b_{i j}=\sum_{j=1}^{t} a_{j}^{(i)} r_{i j} / \sum_{j=1}^{t} a_{j}^{(i)}, \sum_{j=1}^{t} a_{j}^{(i)}=1$.

Namely,

$$
B_{i}=\left(a_{1}^{(i)}, a_{2}^{(i)}, \cdots\right)\left[\begin{array}{ccc}
r_{11} & \cdots & r_{14} \\
\vdots & \ddots & \vdots \\
r_{t 1} & \cdots & r_{t 4}
\end{array}\right]
$$

Finally, based on the second level evaluation, make a comprehensive evaluation to the upper level set $R=\left(B_{1}, B_{2}, \cdots, B_{i}\right)$. The weight value of criterion layer is known as $A=\left\{A_{1}, A_{2}, A_{3}, A_{4}\right\}$, and the total evaluation results vector is shown as follows:

$$
B=A \circ R=\left[A_{1}, A_{2}, A_{3}, A_{4}\right]\left[\begin{array}{ccc}
b_{11} & \cdots & b_{14} \\
\vdots & \ddots & \vdots \\
b_{41} & \cdots & b_{44}
\end{array}\right]=\left[b_{1}, b_{2}, b_{3}, b_{4}\right]
$$

In accordance with the principle of maximum membership degree, if $b_{j}=\max \left\{b_{1}, b_{2}, b_{3}, b_{4}\right\}$, the computer product customer satisfaction belongs to the level of $v_{m}$.

Determine the Degree of Membership. This paper uses binary comparison based on scoring method to determine the sort index membership. For example, set the field as the five factors in product comprehensive performance criterion layer, namely $U_{3}=\left\{u_{1}, u_{2}, u_{3}, u_{4}, u_{5}\right\}$, and $u_{1}$ represents product function, $u_{2}$ represents the hardware configuration, $u_{3}$ represents the software design, $u_{4}$ represents product safety and $u_{5}$ represents product appearance. 10 customers are randomly selected to evaluate each two index of these five index. And select the membership belonging to excellent level. Each expert makes two repeated test in accordance with digital sorting shown in Table. 2, and the results are reported in Table. 3.

Table. 2 Evaluation order of dualistic contrast compositor process based on marking

\begin{tabular}{|c|c|c|c|c|c|}
\hline $\begin{array}{c}\text { Evaluation } \\
\text { order }\end{array}$ & $\mathrm{u} 1$ & $\mathrm{u} 2$ & $\mathrm{u} 3$ & $\mathrm{u} 4$ & $\mathrm{u} 5$ \\
\hline $\mathrm{u} 1$ & & & & & \\
\hline $\mathrm{u} 2$ & 1 & & & & \\
\hline $\mathrm{u} 3$ & 5 & 2 & & & \\
\hline $\mathrm{u} 4$ & 8 & 6 & 3 & & \\
\hline $\mathrm{u} 5$ & 10 & 9 & 7 & 4 & \\
\hline
\end{tabular}

Table. 3 Evaluating results of dualistic contrast compositor process

\begin{tabular}{|c|c|c|c|c|c|c|c|}
\hline $\begin{array}{c}\text { Evaluation } \\
\text { order }\end{array}$ & $\mathrm{u} 1$ & $\mathrm{u} 2$ & $\mathrm{u} 3$ & $\mathrm{u} 4$ & $\mathrm{u} 5$ & Sum & Order \\
\hline $\mathrm{u} 1$ & & 6 & 8 & 5 & 6 & 25 & 2 \\
\hline $\mathrm{u} 2$ & 2 & & 2 & 4 & 3 & 11 & 5 \\
\hline $\mathrm{u} 3$ & 8 & 7 & & 9 & 8 & 31 & 1 \\
\hline $\mathrm{u} 4$ & 3 & 5 & 3 & & 2 & 13 & 4 \\
\hline $\mathrm{u} 5$ & 4 & 6 & 7 & 4 & & 21 & 3 \\
\hline
\end{tabular}

Because the number of total evaluation is 10 , set $A_{1}^{(1)}$ as the membership degree of excellent 
level corresponding to each indexes, and the membership degree value is just the ratio between the preferred number and total number of tests, namely:

$A_{1}^{(1)}=(0.25,0.11,0.31,0.13,0.21)^{T}$

Determine the Weight of Evaluation Indexes. Assign and establish the judgment matrix $C=\left(c_{i j}\right)_{n \times n}$ according to a 1-9 scale method, where the value rule of $c_{i j}$ is shown in Table. 4 .

Table. 4 Evaluating regulation of element $c_{i j}$

\begin{tabular}{|c|c|c|}
\hline Element & Scale degree & Regulation \\
\cline { 2 - 3 } & 1 & The former factor is as important as the latter factor \\
\cline { 2 - 3 }$c_{i j}$ & 3 & The former factor is more important than the latter factor \\
\cline { 2 - 3 } & 5 & The former factor is obviously important than the latter factor \\
\cline { 2 - 3 } & 7 & The former factor is strongly important than the latter factor \\
\cline { 2 - 3 } & $2,4,6,8$ & The former factor is extremely important than the latter factor \\
\hline
\end{tabular}

Normalize the column vector of judgment matrix:

$$
\tilde{C}_{i j}=\left[\frac{C_{i j}}{\sum_{i=1}^{n} c_{i j}}\right]
$$

The feature vector corresponding to the maximum eigenvalue of judgment matrix $\tilde{C}_{i j}$ is as follows:

$$
\tilde{W}=\left[\sum_{j=1}^{n} \frac{c_{1 j}}{\sum_{i=1}^{n} c_{i j}}, \sum_{j=1}^{n} \frac{c_{2 j}}{\sum_{i=1}^{n} c_{i j}}, \cdots \sum_{j=1}^{n} \frac{c_{n j}}{\sum_{i=1}^{n} c_{i j}}\right]^{T}
$$

Normalize $\tilde{W}$, then the weight vector is obtained:

$W=\left(w_{1}, w_{2}, \cdots, w_{n}\right)^{T}$

Fuzzy Comprehensive Evaluation. According to the evaluation index system shown in Table. 1, make first level evaluation for computer product customer satisfaction, the primary single factor fuzzy comprehensive evaluation is:

$$
\begin{aligned}
& B_{1}=\left(\begin{array}{lllll}
\left.a_{1}^{(1)}, a_{2}^{(1)}, a_{3}^{(1)}, a_{4}^{(1)}, a_{5}^{(1)}\right) \\
B_{2}
\end{array}=\left(\begin{array}{llll}
0.300 & 0.340 & 0.320 & 0.004
\end{array}\right)\right. \\
& B_{3}=\left(\begin{array}{llll}
0.205 & 0.313 & 0.349 & 0.083
\end{array}\right)
\end{aligned}
$$

For the target layer, its evaluation index set is $U=\left\{U_{1}, U_{2}, U_{3}\right\}$, construct its judgment matrix:

$$
C=\left[\begin{array}{ccc}
1 & 4 & 2 \\
1 / 4 & 1 & 1 / 2 \\
1 / 2 & 2 & 1
\end{array}\right] \text {. }
$$

Compute the maximum feature vector of judgment matrix $C$ and normalize it, then the weight vector is obtained:

$$
W=\left(\begin{array}{lll}
0.571 & 0.143 & 0.286
\end{array}\right) \text {. }
$$

After calculation, fuzzy comprehensive evaluation model is shown as follows:

$R=\left[B_{1}, B_{2}, B_{3}\right]$

$$
B=W \circ R=\left(\begin{array}{llll}
0.2414 & 0.3169 & 0.3174 & 0.0934
\end{array}\right)
$$

The results show that the membership of computer product customer satisfaction belonging to "excellent", "good", "medium", "bad" are respectively 24.14\%, 31.69\%, 31.74\%, 9.34\%. In accordance with the principle of maximum membership degree, the computer product customer 
satisfaction is "medium" level.

\section{Suggestions on Marketing Countermeasures}

(1) Enhance the overall performance of products. Consumers' demand for computer products has been gradually transferred from the low level of price and function to the higher level of brand, quality and grade. Advanced configuration, excellent quality, convenient use, green environmental protection and other factors have become the hot demand of consumers. Focusing on the comprehensive performance of the products to enhance the company's image and status, which can help to improve the customer satisfaction, and thus enhance the competitiveness of enterprise computer products in the market.

(2) Enhance the brand image of the enterprise. With the advent of the product homogeneity era, brand image becomes one of the important pillars of contemporary enterprises. To enhance the brand image of the enterprise can bring more consumers to the enterprise, so as to have the opportunity to obtain customer satisfaction, thus forming customer loyalty, and finally bring profit to the enterprise. To successfully create the enterprise brand, the computer enterprise should plan the brand image of the enterprise with the international vision, and integrate the planning into the various links of enterprise marketing.

(3) Improve the staff's view of customer satisfaction. Customer satisfaction is not only determined by the management of computer enterprises, but in the service quality and attitude of all employees. At the same time, management is responsible for the behavior and attitude of all employees. Internal employee satisfaction is the premise of external customer satisfaction, and make the enterprise internal employee satisfaction is one of the important measures of establishing staff agree corporate culture, namely establish "maximize customer satisfaction" culture within the enterprise.

\section{Conclusions}

Based on the research of the characteristics of computer products, this paper constructs the evaluation model and evaluation index system of computer product customer satisfaction, evaluates the customer satisfaction of computer products by using fuzzy comprehensive evaluation method, and considers the relevant important factors in the evaluation process. As competition in Chinese computer products market intensifies, competition among enterprises has shifted from product competition to competition of customer resources. To satisfy customers and obtain high customer loyalty is an important magic weapon for Chinese computer enterprises to gain a foothold in domestic and foreign markets.

\section{References}

[1] Cen Y T. Fuzzy Comprehensive Evaluation Model of Customer Satisfaction Evaluation[J]. Journal of Liaoning Technical University, 2001, 20(5): 715-718.

[2] Gao Jinsong, Xu Jinhui, Zhou Zibo, Li Zhoujunl. Customer Satisfaction Evaluation for Mobile Commerce Based on Fuzzy Comprehensive Evaluation[C]// International Symposium on Electronic Commerce and Security. IEEE Computer Society, 2008:679-682.

[3] Lu Jianchang, Han Hongling, Liu Tianbao, Zhao Zhiwei. Comprehensive Evaluation of Power Customer Satisfaction Degree Based on Fuzzy Integral[J]. Power System Technology, 2008, 32(1):67-70.

[4] Zhou Lisha, Yu Xinhua. Fuzzy Comprehensive Evaluation of Power Customer Satisfaction Based on Analytic Network Process[J]. Power System Technology, 2009, 33(17):191-197. 